\title{
Can anesthetic effects and pain treatment influence the long-term prognosis of early-stage lymph node-negative breast cancer after breast-conserving surgery?
}

\author{
Yanan Lu ${ }^{1 \#}$, Ting Liu ${ }^{1 \#}$, Peizong Wang ${ }^{2}$, Yi Chen ${ }^{3}$, Fengtao $\mathrm{Ji}^{1}$, Fernando Hernanz ${ }^{4}$, Gustavo \\ Zucca-Matthes $^{5}$, Sherif Youssif ${ }^{6,7}$, Shuling Peng ${ }^{1}$, Dongni Xu ${ }^{1}$
}

${ }^{1}$ Department of Anesthesiology, Sun Yat-sen Memorial Hospital, Sun Yat-sen University, Guangzhou, China; ${ }^{2}$ Department of Anesthesiology, Sun Yat-sen University Cancer Center, Guangzhou, China; ${ }^{3}$ Su Fengxi Clinic, Guangzhou, China; ${ }^{4}$ Oncoplastic Breast Unit, Hospital Universitario Valdecilla, University of Cantabria, Santander, Spain; ${ }^{5}$ Department of Gynaecology, Obstetrics and Mastology, School of Medicine of Botucatu, UNESP,Botucatu-SP, Brazil; ${ }^{6}$ Plastic and Reconstructive Surgery Division, Good Hope Hospital, University Hospitals Birmingham NHS Trust, Birmingham, UK; ${ }^{7}$ Plastic surgery department, Assiut University, Assiut, Egypt

Contributions: (I) Conception and design: D Xu, Y Lu, S Peng; (II) Administrative support: D Xu, Y Lu; (III) Provision of study materials or patients: Y Chen, F Ji, D Xu; (IV) Collection and assembly of data: T Liu, Y Lu, D Xu; (V) Data analysis and interpretation: D Xu, T Liu, Y Lu; (VI) Manuscript writing: All authors; (VII) Final approval of the manuscript: All authors.

\#These authors contributed equally to this work and should be considered co-first authors.

Correspondence to: Dongni Xu, MD; Shuling Peng, MD, PhD. Department of Anesthesiology, Sun Yat-sen Memorial Hospital, Sun Yat-sen University, Guangzhou, China. Email: xudongni@mail.sysu.edu.cn; pslmzk@aliyun.com.

\begin{abstract}
Background: Breast cancer is currently the leading cause of women's death. It is crucial to further improve the approach to treatment and the long-term survival rate of breast cancer patients, and to reduce the rates of recurrence and metastasis. It has been reported that the possibility of tumor metastasis depends on the metastatic potential of the tumor and the host defense against tumor metastasis, in which cellular immunity and the function of natural killer (NK) cells are critical to maintaining this balance. Surgical stress response and postoperative pain inhibit perioperative immune function in patients and increase the likelihood of dissemination and metastasis of cancer cells after cancer surgery. The study aims to investigate the effect of anesthetic factors and pain treatment on the long-term prognosis of patients with early stage lymph node negative breast preservation surgery.

Methods: A total of 337 patients with early-stage lymph node negative breast cancer (ASA I-II) who had undergone successful breast-conserving surgery in our hospital were included in this retrospective analysis. Cases were divided into general anesthesia with postoperative analgesia group (GA + PCA), general anesthesia without postoperative analgesia group (GA), epidural anesthesia with postoperative analgesia group (EA + PCA), and epidural anesthesia without postoperative analgesia group (EA). The 5-year survival rate and 5-year disease-free survival were recorded in the 4 groups.

Results: The general condition and length of hospital stay of the patients were not statistically different between the 4 groups. However, the 5 -year survival rate and 5-year disease-free survival rate of the 4 groups were statistically different. The 5-year survival rate and 5-year disease-free survival rate were the lowest in the GA group, while the EA + PCA group had the highest 5-year disease-free survival rate. The 5-year survival rate and 5-year disease-free survival rate in the GA + PCA group were significantly higher than those in the GA group. The 5-year disease-free survival rate in EA group was significantly higher than GA group. Conclusions: Epidural anesthesia and postoperative pain treatment maybe beneficial to the long-term prognosis of patients with early-stage lymph node-negative breast cancer.
\end{abstract}

Keywords: General anesthesia; epidural anesthesia; pain; breast cancer; metastasis; recurrence

Submitted Apr 07, 2021. Accepted for publication Sep 10, 2021.

doi: $10.21037 / \mathrm{atm}-21-4392$

View this article at: https://dx.doi.org/10.21037/atm-21-4392 


\section{Introduction}

Breast cancer accounts for the highest proportion of women's deaths in the world (1). Therefore, it is critical to improve the cure rate and long-term survival rate of breast cancer patients, and reduce the rate of recurrence and metastasis. It has been reported that the possibility of tumor metastasis depends on the tumor metastasis potential and the host defense against tumor metastasis, in which cellular immunity and natural killer (NK) cell function are essential to maintain this balance (2). For patients with breast cancer, breast preservation surgery, which includes resection of the tumor and surrounding tissues, biopsy or dissection of the ipsilateral axillary lymph nodes, is both a treatment process and body damage. Severe stress response and postoperative pain inhibit patient perioperative immune function and increase the possibility of cancer cell dissemination and metastasis after cancer surgery. Large doses of opioids have been reported to induce immunosuppression in cell culture experiments and animal models $(3,4)$. However, some researchers believe that opioids during surgery improve disease-free survival of triple negative breast cancer (5), and spinal anesthesia and local anesthesia can prevent immunosuppression during the perioperative period (6). Other perioperative conditions, including blood transfusions, pain, stress, and hypothermia, are also potentially important factors interfering with perioperative immunity (2).

General anesthesia can inhibit the limbic system of the cerebral cortex or the hypothalamus projection system to the cerebral cortex. However, it cannot effectively block the conduction pathway of nociceptive stimulation to the center of the surgical area. General anesthesia may also affect neutrophil, macrophage, and dendritic T cellfunction while inhibiting cell immunity (7). However, an epidural block can suppress the spinal cord up nerve impulse generated by surgical stimulation, reduce its effect on the hypothalamuspituitary-adrenal cortex axis, and inhibit sympathetic nerve activity. It also does not affect the sensitivity of the immune response, which to some extent alleviates the cellular immunosuppression caused by surgical stress (7). The epidural block of the high thoracic segment blocks the central nervous system's ability to sense nociceptive stimulation, blocks the harmful stimulation of $\mathrm{A} \sigma$ and $\mathrm{C}$ fiber conduction, inhibits the sympathetic nervous system, and reduces the release of adrenaline from the medulla (8).

Postoperative pain is critical for the prognosis of patients. It directly affects multiple systems and causes physiological, immunological, and psychological changes (9). Acute pain inhibits NK cell activity (10). Excellent postoperative analgesia can reduce surgical stressinducedimmunosuppression and improve the long-term survival rate of patients with malignant tumors (11).

Therefore, perioperative anesthesia management in cancer patients may affect prognosis and recurrence (12). Choosing the appropriate anesthetic method and pain treatment, and controlling a series of pathophysiological changes after perioperative stress response to a reasonable range, has become a skill essential for anesthesiologists to acquire. The original meaning of anesthesia refers to the loss of sensation and perception, but now includes meanings related to a state in which the patient does not feel pain or discomfort during surgery or invasive operation. The goal of anesthesia has also changed from pain relief to maintaining the patient's recovery and regeneration ability as much as possible.

Hence, we first of its kind to selected successful breast conserving patients with early-stage lymph node-negative , and explored the effects of anesthesia and pain treatment on the long-term prognosis. We present the following article in accordance with the STROBE reporting checklist (available at https://dx.doi.org/10.21037/atm-21-4392).

\section{Methods}

\section{Case selection}

All procedures performed in this study involving human participants were in accordance with the Declaration of Helsinki (as revised in 2013). The study was approved by institutional ethics committee of Sun Yat-sen Memorial Hospital (No.: SYSEC-KY-KS-2021-078) and individual consent for this retrospective analysis was waived. After receiving approval from the local ethics committee, we consulted the data of more than 2,000 patients who underwent breast surgeries at Sun Yat-sen Memorial Hospital between 2004 and 2011. The five-year survival rate and five-year disease-free survival rate were selected as the main survival indicators of this study, so the followup time of the retrospective cases should exceed five years as far as possible. In order to collect enough cases that meet the inclusion and exclusion criteria, we selected the retrospective analysis of the cases from 2004 to 2011. And we homogenized other influencing factors as much as possible, according to the selection criteria and exclusion criteria, 337 patients were included in this study. 


\section{Selection criteria}

The inclusion criteria for patients in this study were the following: American Society of Anesthesiology (ASA) grade I-II; aged 18-70 years; female; pathological tumorstage T1-2N0M0 (tumor size less than $5 \mathrm{~cm}$ in diameter, resectable, and no lymph node or distant metastasis); lymph node-negative; history of standardized chemotherapy; chemotherapy of CMF (cyclophosphamide + methotrexate +5 -fluorouracil), CAF (cyclophosphamide + doxorubicin +5 -fluorouracil), or CEF (cyclophosphamide + epirubicin +5 -fluorouracil); and radiotherapy and endocrine therapy (tamoxifen or the same type of Fallot, toremifene, and raloxifene) were performed after operation. The surgical approach was required to be breast-conserving surgery, with the anesthetic method being general inhalation anesthesia or epidural anesthesia.

\section{Exclusion criteria}

The exclusion criteria for patients were the following: a history of intraoperative or postoperative blood transfusion; older than 70 years or younger than 18 years; male; inconsistent pathological stage of the tumor; lymph node metastasis positive; the presence of rare histopathological subtypes (malignant phyllodes tumor, angiosarcoma, salivary adenoid tumor, neuroendocrine carcinoma, and metaplastic carcinoma); triple-negative breast cancer (i.e., human epidermal growth factor receptor 2 and estrogen receptor-negative by immunohistochemistry, and negative for progesterone receptor); history of unsuccessful breastconserving surgery; poor anesthetic effect, including malignant hypertension or continuous hypotension during operation; delayed postoperative recovery, intraoperative awareness, postoperative bleeding, wound infection, and other complications; and pregnant women with previous tumor history.

The 337 patients were included in the retrospective study signed the informed consent prior to surgery and for anesthesia.

\section{Case information}

Among the 337 patients reviewed in the study, there were 80 patients under epidural anesthesia and 257 under general anesthesia.

\section{Epidural anesthesia}

These 80 patients had a good effect of epidural anesthesia, and there were no patients who needed to change to general endotracheal anesthesia. According to our hospital regulations, high thoracic epidural anesthesia was operated by the attending physician or above. Usually, thoracic vertebra clearance $3-4$ or $4-5$ was chosen, and an $18-G$ epidural puncture needle was used. The test dose was $3 \mathrm{~mL}$ of $1 \%$ lidocaine, and $0.3-0.5 \%$ ropivacaine was used during the operation; the total volume was between 12 and $18 \mathrm{~mL}$. No severe complications, including respiratory depression, nerve injury, total spinal anesthesia, etc. occurred in these patients. Sixty-six patients needed compound fenflurane mixture or duflurane mixture, and five patients needed compound ketamine.

\section{General anesthesia}

Two hundred and seventy-seven patients under general anesthesia received midazolam $1 \mathrm{mg}$, propofol $1-2 \mathrm{mg} / \mathrm{kg}$, fentanyl 3-5 mg/kg, and muscle relaxant. Sevoflurane or desflurane between 0.8 and 1.3 MAC (minimum alveolar concentration) was used for intraoperative maintenance. There were no recovery delays, intraoperative awareness, and postoperative cognitive impairment in these cases.

\section{Analgesia}

One hundred and sixty-eight patients used a postoperative intravenous analgesia pump for postoperative analgesia. The analgesic pump was continuous infusion $(2 \mathrm{~mL} / \mathrm{h})$, and the analgesic was flurbiprofen or lornoxicam.

\section{Surgical program and other adjuvant treatment schemes}

All the cases included in the study were patients who successfully underwent breast-conserving surgery. The same surgical team completed the operations. Furthermore, the same team performed standardized chemotherapy, radiotherapy, and endocrine therapy after the operation in all cases reviewed.

According to the patient's anesthesia methods and analgesia program, patients were divided into epidural anesthesia with postoperative Patient-controlled analgesia group (EA + PCA group), and epidural anesthesia group without postoperative analgesia (EA group), general anesthesia with postoperative Patient-controlled analgesia group (GA + PCA group), and a general anesthesia group without postoperative analgesia (GA group).

\section{Analytical indicators}

The primary indicators included the 5-year survival rate 
Table 1 Characteristics of 337 patients in the 4 groups

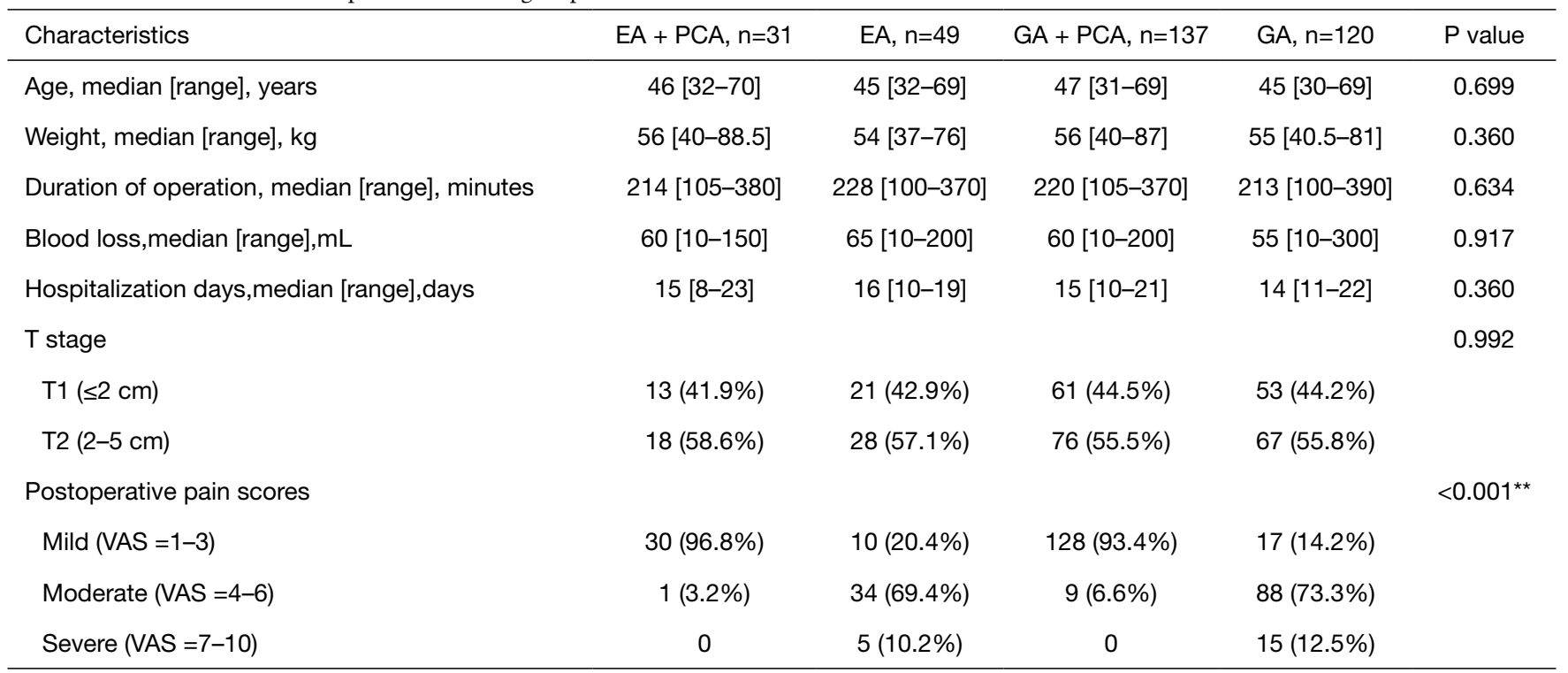

**, indicates a statistically significant difference $(P<0.01)$. $E A+P C A$, epidural anesthesia with postoperative analgesia group; $E A$, epidural anesthesia without postoperative analgesia group; GA + PCA, general anesthesia with postoperative analgesia group; GA, general anesthesia without postoperative analgesia group; VAS, visual analogue scales.

and the 5-year disease-free survival rate. Records of patients with local recurrence, contralateral recurrence, secondary cancer, bone metastasis, liver metastasis, brain metastasis, and lymph node metastasis were also considered. The secondary indicator was the number of days in the hospital.

General information incorporated into our analysis included body weight, age, operation time, and blood loss.

\section{Postoperative follow-up}

All patients included in the retrospective analysis were followed up by a full-time breast surgeon at Sun Yat-sen Memorial Hospital. The doctors did not participate in the patient's operation plan or another treatment plan. The postoperative follow-up period was more than 5 years.

\section{Statistical analysis}

All data collected were recorded in a Microsoft Excel spreadsheet and imported into SPSS 19.0 statistical software (IBM Corp.) statistical software for analysis. Measurement data are expressed as mean \pm standard deviation (s). Singlefactor analysis of variance (ANOVA) was used after the regular test and variance homogeneity test. Two tests were used to compare the count data. When there was a statistical difference in the count data between the groups, the statistics were further compared pairwise, and the Bonferroni method was used to correct the $\alpha$ value, with $\mathrm{P}<\alpha$ considered statistically significant. Furthermore, the survival curve and the risk factor curve of the 5-year survival rate and the 5-year disease-free survival rate were made for the 4 groups; the log-rank test was used for further pairwise comparison. $\mathrm{P}$ value $<0.05$ indicated statistical significance.

\section{Results}

\section{General data comparison of the 4 groups}

After the selection and exclusion criteria, 337 patients who underwent breast preservation surgery were evaluated (Table 1). Age, body weight, operation time, and blood loss were not significantly different between the 4 groups $(\mathrm{P}>0.05)$, as shown in Table 1.

\section{Length of stay comparison between the 4 groups}

There were no significant differences in stay duration between the 4 groups $(\mathrm{P}>0.05)$, as shown in Table 1 .

\section{Pain comparison between the 4 groups}

Pain evaluation of the 337 patients consisted of 3 levels: 
Table 2 Five-year survival rate survival rate and five-year disease-free survival rate of 337 patients in the 4 groups

\begin{tabular}{|c|c|c|c|c|c|}
\hline Outcome & $E A+P C A, n=31(\%)$ & $E A, n=49(\%)$ & $\mathrm{GA}+\mathrm{PCA}, \mathrm{n}=137(\%)$ & $\mathrm{GA}, \mathrm{n}=120(\%)$ & $P$ value \\
\hline 5-year disease-free survival rate & $30(96.8)$ & 47 (95.9) & $128(93.4)$ & $95(79.2)$ & $<0.001^{\star *}$ \\
\hline
\end{tabular}

Table 3 Details of recurrence, metastasis, and second cancer of 337 patients in the 4 groups

\begin{tabular}{|c|c|c|c|c|}
\hline Characteristic & $\mathrm{EA}+\mathrm{PCA}, \mathrm{n}=31(\%)$ & $E A, n=49(\%)$ & $\mathrm{GA}+\mathrm{PCA}, \mathrm{n}=137(\%)$ & $\mathrm{GA}, \mathrm{n}=120(\%)$ \\
\hline \multicolumn{5}{|l|}{ Recurrence } \\
\hline Local recurrence & $1(3.2)$ & 0 & 0 & $10(8.3)$ \\
\hline Recurrence on the other side & 0 & $1(2.0)$ & $1(0.7)$ & 0 \\
\hline The second cancer & 0 & $1(2.0)$ & $1(0.7)$ & 0 \\
\hline Liver metastasis & 0 & 0 & 0 & $5(4.2)$ \\
\hline Bonemetastasis & 0 & 0 & $3(2.2)$ & $5(4.2)$ \\
\hline Lung metastasis & 0 & 0 & $3(2.2)$ & $6(73.3)$ \\
\hline Brain metastasis & 0 & 0 & 0 & $2(1.7)$ \\
\hline
\end{tabular}

$\mathrm{EA}+\mathrm{PCA}$, epidural anesthesia with postoperative analgesia group; EA, epidural anesthesia without postoperative analgesia group; GA + PCA, general anesthesia with postoperative analgesia group; GA, general anesthesia without postoperative analgesia group.

mild, moderate, and severe. VAS score of $1-3$ was considered mild pain, 4-6 was considered moderate pain, and 7-10 was considered severe pain. According to this classification, a significant difference in pain was observed between the 4 groups $(\mathrm{P}<0.001)$, as shown in Table 1 .

\section{Long-term survival comparison between the 4 groups}

The 5 -year survival rate of the 4 groups was significantly different $(\mathrm{P}=0.018)$, as was the 5 -year disease-free survival rate $(\mathrm{P}<0.001)$, as shown in Table 2. Details of postoperative recurrence, metastasis, and second cancer in the 4 groups are shown in Table 3; the cumulative survival curves and logarithmically transformed hazard function curves are shown in Figure 1; the disease-free survival curves and logarithmically transformed hazard function curves are shown in Figure 2.

The cumulative survival rate of the 4 groups was analyzed by survival curves and logarithmically transformed hazard function curves (Log-rank test was used, $\mathrm{P}=0.033$ ). This indicated that the cumulative survival rate curves and logarithmically transformed hazard function curves were not identical, and survival curves analyzed the disease-free survival rate and logarithmically transformed hazard function curves (Log-rank test was used, $\mathrm{P}=0.002$ ). This indicated that the survival rate curves and logarithmically transformed hazard function curves were not identical. Log-rank test was used for further pairwise comparison, as shown in Table 4.

A combination of the survival curve and the logarithmic transformed hazard function curve revealed that the cumulative overall survival rate and disease-free survival rate of the GA group were the lowest, while the disease-free survival rate of the EA + PCA group was the highest. The cumulative overall survival rate and disease-free survival rate of the GA + PCA group were higher than those of the GA group $(\mathrm{P}=0.008$, $\mathrm{P}=0.004)$. The disease-free survival rate of the $\mathrm{EA}$ group was higher than the GA group $(\mathrm{P}=0.011)$, as shown in Table 4.

\section{Discussion}

It remains unclear whether the anesthetic effect can 

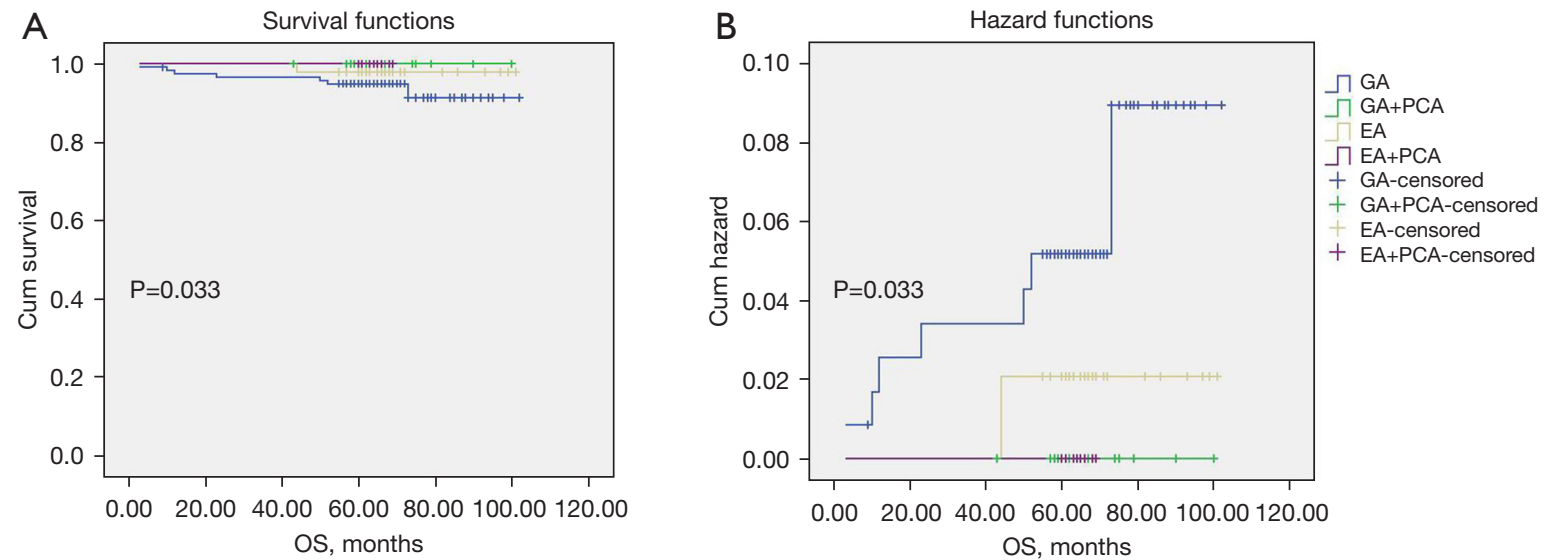

Figure 1 Cumulative overall survival curves for 337 patients who were underwent breast-conserving surgery operation cancer divided into EA + PCA group, EA group, GA + PCA group, GA group. EA + PCA, epidural anesthesia with postoperative analgesia group; EA, epidural anesthesia without postoperative analgesia group; GA + PCA, general anesthesia with postoperative analgesia group; GA, general anesthesia without postoperative analgesia group.
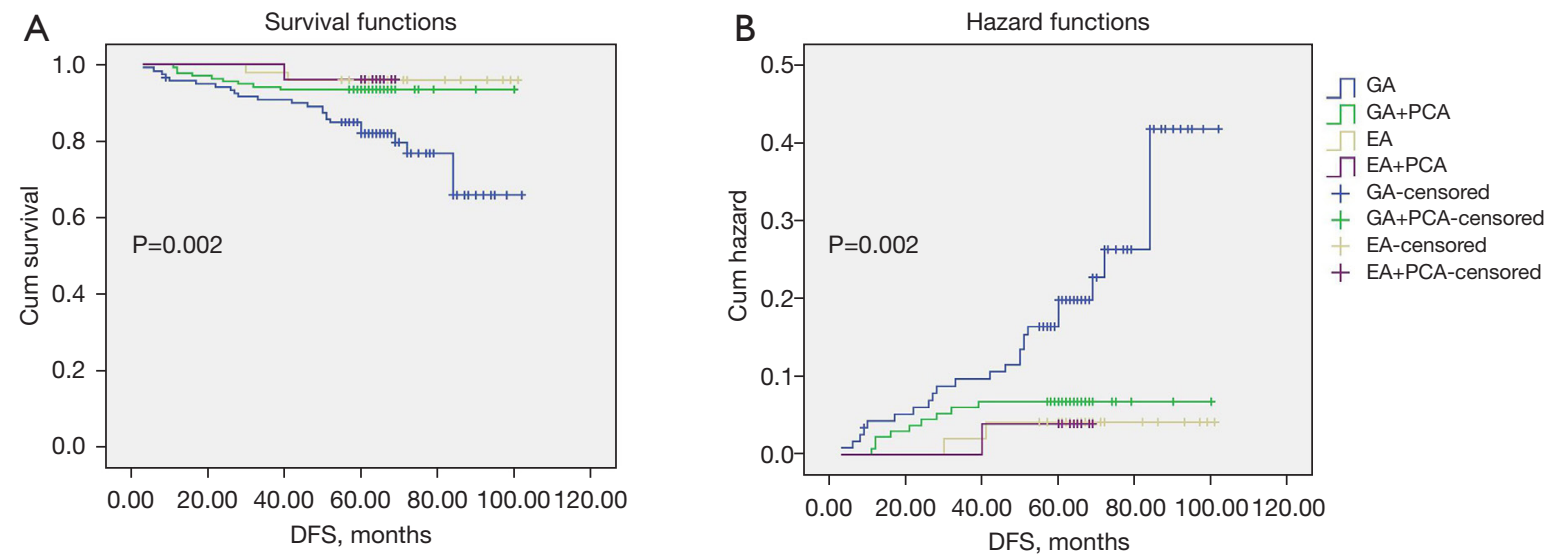

Figure 2 Disease-free survival curves for 337 patients who were underwent breast-conserving surgery operation cancer divided into EA + PCA group, EA group, GA + PCA group, GA group. EA + PCA, epidural anesthesia with postoperative analgesia group; EA, epidural anesthesia without postoperative analgesia group; GA + PCA, general anesthesia with postoperative analgesia group; GA, general anesthesia without postoperative analgesia group.

Table 4 Comparison of log-rank test for overall survival and disease-free survival in the 4 groups

\begin{tabular}{lcc}
\hline Groups & OS, P value & DFS, P value \\
\hline EA + PCA/EA group & 0.466 & 0.961 \\
EA + PCA/GA + PCA group & $※$ & 0.584 \\
EA/GA group & 0.325 & $0.011^{*}$ \\
GA + PCA/GA group & $0.008^{\star *}$ & $0.004^{* *}$
\end{tabular}

※, cannot be compared; *, indicates a statistically significant difference $(\mathrm{P}<0.05)$; ${ }^{\star \star}$, indicates a statistically significant difference $(\mathrm{P}<0.01)$. OS, overall survival; DFS, disease-free survival; EA + PCA, epidural anesthesia with postoperative analgesia group; EA, epidural anesthesia without postoperative analgesia group; GA + PCA, general anesthesia with postoperative analgesia group; GA, general anesthesia without postoperative analgesia group. 
influence the long-term prognosis of patients with malignant tumors. Satisfactory anesthesia should relieve pain, provide muscle relaxation, ensure that the patient is safe during the perioperative period, and maintain the recovery and regeneration ability of patients as much as possible. Perioperative management profoundly impacts cancer and its treatment; the best perioperative management requires an individualized assessment of the impact and treatment of cancer on the functional reserve of the primary organ system (13). Breast cancer is a heterogeneous disease, and the immune response degree of regulating tumor growth varies with the pathological stages and pathological subtypes. Surgical treatment (including modified radical operation and breast preservation surgery) is the approach to breast cancer treatment. However, the operation is influenced by neuroendocrinal, metabolic, and cytokine responses, affecting clinical outcomes. A previous retrospective analysis found that anesthesia and optimal postoperative pain treatment could help improve the 5-year survival rate and the 5-year disease-free survival rate of patients with earlystage lymph node-negative breast patients who underwent breast-conserving surgery (14). A common view is that the tumor recurrence rate can potentially correlate with the stress of operation itself. General anesthesia, opioid drugs, regional anesthesia, and postoperative analgesia are beneficial in reducing the rate of tumor recurrence. However, these conclusions are also controversial. Our study was the first of its kind to explore the effect of anesthesia and pain treatment on the prognosis of patients with early-stage lymph node-negative breast cancer who had undergone breast preservation surgery. In this study, to balance the primary data of the 4 groups of patients, we used rigorous inclusion criteria and exclusion criteria to eliminate the interference of the breast cancer disease itself (the size of the primary tumor, pathological classification, lymph node condition, etc. can affect prognosis) and treatment methods.

\section{Perioperative factors and long-term prognosis of breast cancer}

The perioperative period consists of the entire operation, including preoperative, intraoperative, and postoperative stages. Preoperative anesthesia and drug choice directly impact perioperative regulation of immune function. Several factors in the perioperative period will affect tumor cell recurrence and metastasis, including antitumor cell immunity and signaling in promoting tumor cell angiogenesis and growth (15). Perioperative anesthesia factors can affect long- term tumor recurrence and metastasis, and its mechanism may be related to perioperative immunosuppression and the release of vascular endothelial growth factor (VEGF) and transforming growth factor $\beta$ (TGF- $\beta$ ) $(16,17)$. Many studies suggest that perioperative anesthesia and drug selection directly impact the regulation of immune function. Most inhaled anesthetics (e.g., halothane, enflurane, and nitrous oxide), intravenous general anesthetics, (e.g., thiopental, ketamine, and morphine analgesics), and local anesthetics can inhibit cellular immunity, and affect lymphocyte DNA synthesis, differentiation, and transformation. The decrease in the number and the absolute value of the subsets of $\mathrm{T}$ lymphocytes is a critical manifestation of postoperative immunosuppression, and the degree of decrease is consistent with the degree of immunosuppression (18). Anesthetics can also act directly on NK cells and inhibit NK cell activity during the perioperative period (19). Therefore, several factors in the perioperative period may affect the treatment of patients with breast cancer: the operation itself, perioperative surgical stress, the method and drugs of anesthesia, and other factors such as blood transfusions, pain, and hypothermia (2).

\section{Effect of general anesthesia on the long-term prognosis of patients with breast cancer}

General anesthesia can inhibit the cortical, limbic, or hypothalamic projection system to the cerebral cortex, but it cannot effectively block the transmission pathway of noxious stimulation to the central nervous system. Some literature indicates that general anesthesia inhibits cellular immunity and damages the functions of neutrophils, macrophages, and dendritic T cells (11).

Several studies have reported that the serum level of VEGF-C in patients undergoing breast cancer surgery and general anesthesia is higher than in patients undergoing paravertebral block, but the level of TGF- $\beta$ is lower; these changes may be adverse to the long-term prognosis of cancer patients (8). Inhalation of general anesthetics can inhibit NK cell activity by inhibiting interferon production, increasing the probability of metastasis and tumor cell recurrence (6). Sevoflurane may also increase the risk of new malignant diseases in cancer patients up to 5 years after surgery (3).

\section{Effect of intraspinal anesthesia and regional anesthesia on the long-term prognosis of patients with breast cancer}

Epidural block can suppress the spinal cord's nerve impulse, reduce its influence on the hypothalamic-pituitary-adrenal 
cortex axis, and inhibit sympathetic nerve activity. However, epidural anesthesia does not affect sensitivity to the immune response, which alleviates cellular immunosuppression caused by surgical stress to some extent (11). Studies have shown that regional blockage and analgesia do not affect VEGF and PGE2 levels after breast cancer surgery (20). Animal models have also shown that regional block anesthesia is better than general anesthesia for NK cell function and reduces distant metastasis and local recurrence $(21,22)$.

\section{Effect of pain therapy on the long-term prognosis of breast cancer patients}

Postoperative pain is critical for the prognosis of patients. It directly affects multiple systems and causes physiological, immunological, and psychological changes (23). Acute pain inhibits NK cell activity (10). A model of tumor cell metastasis model in vitro showed that acute postoperative pain caused the release of substance $\mathrm{P}$, which was involved in pain conduction and mediated neurogenic inflammation after binding to NK-1 receptors, and promoted distant migration of breast cancer cells (24).

The effect of perioperative opioids on immune function is a complex problem. In addition to cellular immunity, opioids also inhibit humoral immunity during the perioperative period (25). However, under perioperative surgical stress, opioids can alleviate immunosuppression caused by surgical trauma through analgesic effects. Animal studies have shown that opioids can relieve immunosuppression caused by surgical trauma in rats by suppressing pain (26).

Perioperative use of non-steroidal anti-inflammatory drugs may be beneficial for 5 -year survival in patients with breast cancer, as they can suppress prostaglandin synthesis by inhibiting cyclooxygenase. Interestingly, one of the mechanisms by which cancer patients escape cellular immunity is by releasing prostaglandin (27).

\section{Limitations}

This is a single center retrospective study which only describes the possible impacts of anesthesia and pain treatment on breast cancer survival, so more research is needed to make more depth analyses. The outcome in our study maybe just a starting point to plan prospective well-designed randomized controlled studies to prove if anesthetic types have some effects on oncologic long-term prognosis in breast cancer.

\section{Conclusions}

In this study, the statistical results of the survival analysis showed that in patients without postoperative pain treatment, the 5-year survival rate and the 5-year diseasefree survival rate of the epidural group were higher than those of the general anesthesia group, and that there was a statistical difference in the 5 -year disease-free survival rate between the two groups. We further found that the 5-year and 5-year disease-free survival rates of general anesthesia patients with postoperative pain treatment were higher than those of the GA group, and the difference was statistically significant.

Therefore, compared to general anesthesia, intraspinal anesthesia, and postoperative analgesia have a lower risk of cancer recurrence and metastasis. Considering the difficulty and risk of spinal anesthesia, a regional block may be a feasible solution that warrants further investigation. The array of pectoral nerve blocks (PECS) maybe a good choice. The PECS block can provide more effective analgesia for patients with breast cancer surgery and decrease an additional analgesic (28), especially those who underwent general anesthesia. Compared to other regional techniques, the PECS block may also perform better, for the included 3 types (PECS I, PECS II, and serratus plane blocks) (28).

\section{Acknowledgments}

The authors appreciate the academic support from the AME Breast Cancer Collaborative Group and Drs. Quanyuan Shan, Fengxi Su, and Oscar J. Manrique.

Funding: Natural Science Foundation of Guangdong Province (2021A1515012357).

\section{Footnote}

Reporting Checklist: The authors have completed the STROBE reporting checklist. Available at https://dx.doi. org/10.21037/atm-21-4392

Data Sharing Statement: Available at https://dx.doi. org/10.21037/atm-21-4392

Conflicts of Interest: All authors have completed the ICMJE uniform disclosure form (available at https://dx.doi. org/10.21037/atm-21-4392). The authors have no conflicts 
of interest to declare.

Ethical Statement: The authors are accountable for all aspects of the work in ensuring that questions related to the accuracy or integrity of any part of the work are appropriately investigated and resolved. All procedures performed in this study involving human participants were in accordance with the Declaration of Helsinki (as revised in 2013). The study was approved by institutional ethics committee of Sun Yat-sen Memorial Hospital (No.: SYSEC-KY-KS-2021-078) and individual consent for this retrospective analysis was waived.

Open Access Statement: This is an Open Access article distributed in accordance with the Creative Commons Attribution-NonCommercial-NoDerivs 4.0 International License (CC BY-NC-ND 4.0), which permits the noncommercial replication and distribution of the article with the strict proviso that no changes or edits are made and the original work is properly cited (including links to both the formal publication through the relevant DOI and the license). See: https://creativecommons.org/licenses/by-nc-nd/4.0/.

\section{References}

1. Karanlik H, Kılıç B, Yıldırım I, et al. Breast-Conserving Surgery Under Local Anesthesia in Elderly Patients with Severe Cardiorespiratory Comorbidities: A Hospital-Based Case-Control Study. Breast Care (Basel) 2017;12:29-33.

2. Snyder GL, Greenberg S. Effect of anaesthetic technique and other perioperative factors on cancer recurrence. Br J Anaesth 2010;105:106-15.

3. Lindholm ML, Granath F, Eriksson LI, et al. Malignant disease within 5 years after surgery in relation to duration of sevoflurane anesthesia and time with bispectral index under 45. Anesth Analg 2011;113:778-83.

4. Gottschalk A, Sharma S, Ford J, et al. Review article: the role of the perioperative period in recurrence after cancer surgery. Anesth Analg 2010;110:1636-43.

5. Montagna G, Gupta HV, Hannum M, et al. Intraoperative opioids are associated with improved recurrence-free survival in triple-negative breast cancer. Br J Anaesth 2021;126:367-76.

6. Kim R, Kawai A, Wakisaka M, et al. Outpatient breastconserving surgery for breast cancer: Use of local and intravenous anesthesia and/or sedation may reduce recurrence and improve survival. Ann Med Surg (Lond) 2020;60:365-71.
7. Deegan CA, Murray D, Doran P, et al. Anesthetic technique and the cytokine and matrix metalloproteinase response to primary breast cancer surgery. Reg Anesth Pain Med 2010;35:490-5.

8. Christopherson R, James KE, Tableman M, et al. Longterm survival after colon cancer surgery: a variation associated with choice of anesthesia. Anesth Analg 2008;107:325-32.

9. Kehlet H, Holte K. Effect of postoperative analgesia on surgical outcome. Br J Anaesth 2001;87:62-72.

10. Page GG. Acute pain and immune impairment. Pain Clin Updates 2005;13:1-4.

11. Yardeni IZ, Beilin B, Mayburd E, et al. The effect of perioperative intravenous lidocaine on postoperative pain and immune function. Anesth Analg 2009;109:1464-9.

12. Arain MR, Buggy DJ. Anaesthesia for cancer patients. Curr Opin Anaesthesiol 2007;20:247-53.

13. Dubowitz JA, Sloan EK, Riedel BJ. Implicating anaesthesia and the perioperative period in cancer recurrence and metastasis. Clin Exp Metastasis 2018;35:347-58.

14. Sessler DI. Does regional analgesia reduce the risk of cancer recurrence? A hypothesis. Eur J Cancer Prev 2008;17:269-72.

15. Sessler DI, Ben-Eliyahu S, Mascha EJ, et al. Can regional analgesia reduce the risk of recurrence after breast cancer? Methodology of a multicenter randomized trial. Contemp Clin Trials 2008;29:517-26.

16. Rocca A, Cancello G, Bagnardi V, et al. Perioperative serum VEGF and extracellular domains of EGFR and HER2 in early breast cancer. Anticancer Res 2009;29:5111-9.

17. Looney M, Doran P, Buggy DJ. Effect of anesthetic technique on serum vascular endothelial growth factor $\mathrm{C}$ and transforming growth factor $\beta$ in women undergoing anesthesia and surgery for breast cancer. Anesthesiology 2010;113:1118-25.

18. Soerjomataram I, Louwman MW, Ribot JG, et al. An overview of prognostic factors for long-term survivors of breast cancer. Breast Cancer Res Treat 2008;107:309-30.

19. Volk T, Schenk M, Voigt K, et al. Postoperative epidural anesthesia preserves lymphocyte, but not monocyte, immune function after major spine surgery. Anesth Analg 2004;98:1086-92.

20. O'Riain SC, Buggy DJ, Kerin MJ, et al. Inhibition of the stress response to breast cancer surgery by regional anesthesia and analgesia does not affect vascular endothelial growth factor and prostaglandin E2. Anesth Analg 2005;100:244-9. 


\section{Page 10 of 10}

21. Wada H, Seki S, Takahashi T, et al. Combined spinal and general anesthesia attenuates liver metastasis by preserving TH1/TH2 cytokine balance. Anesthesiology 2007;106:499-506.

22. Bar-Yosef S, Melamed R, Page GG, et al. Attenuation of the tumor-promoting effect of surgery by spinal blockade in rats. Anesthesiology 2001;94:1066-73.

23. Tedore $T$. Regional anaesthesia and analgesia: relationship to cancer recurrence and survival. Br J Anaesth 2015;115 Suppl 2:ii34-45.

24. Muñoz M, Rosso M, Aguilar FJ, et al. NK-1 receptor antagonists induce apoptosis and counteract substance P-related mitogenesis in human laryngeal cancer cell line HEp-2. Invest New Drugs 2008;26:111-8.

Cite this article as: Lu Y, Liu T, Wang P, Chen Y, Ji F, Hernanz F, Zucca-Matthes G, Youssif S, Peng S, Xu D. Can anesthetic effects and pain treatment influence the long-term prognosis of early-stage lymph node-negative breast cancer after breast-conserving surgery? Ann Transl Med 2021;9(18):1467. doi: $10.21037 / \mathrm{atm}-21-4392$

\section{Lu et al. Can anaesthesia and pain affect breast cancer}

25. Ecimovic P, Murray D, Doran P, et al. Direct effect of morphine on breast cancer cell function in vitro: role of the NET1 gene. Br J Anaesth 2011;107:916-23.

26. Page GG, Blakely WP, Ben-Eliyahu S. Evidence that postoperative pain is a mediator of the tumor-promoting effects of surgery in rats. Pain 2001;90:191-9.

27. Harris RE, Beebe-Donk J, Alshafie GA. Reduction in the risk of human breast cancer by selective cyclooxygenase- 2 (COX-2) inhibitors. BMC Cancer 2006;6:27.

28. Ueshima H, Otake H, Hara E, et al. How to Use Pectoral Nerve Blocks Effectively_An Evidence-Based Update Asian J Anesthesiol 2019;57:28-36.

(English Language Editors: J. Gray and J. Chapnick) 